\title{
TERAHERTZ SENSING TECHNOLOGY
}




\section{SELECTED TOPICS IN ELECTRONICS AND SYSTEMS}

\section{Editor-in-Chief: $\quad$ M. S. Shur}

\section{Published}

Vol. 15: Silicon and Beyond eds. M. S. Shur and T. A. Fjeldly

Vol. 16: Advances in Semiconductor Lasers and Applications to Optoelectronics eds. M. Dutta and M. A. Stroscio

Vol. 17: Frontiers in Electronics: From Materials to Systems eds. Y. S. Park, S. Luryi, M. S. Shur, J. M. Xu and A. Zaslavsky

Vol. 18: Sensitive Skin eds. V. Lumelsky, M. S. Shur and S. Wagner

Vol. 19: Advances in Surface Acoustic Wave Technology, Systems and Applications (Two volumes), volume 1 eds. C. C. W. Ruppel and T. A. Fjeldly

Vol. 20: Advances in Surface Acoustic Wave Technology, Systems and Applications (Two volumes), volume 2 eds. C. C. W. Ruppel and T. A. Fjeldly

Vol. 21: High Speed Integrated Circuit Technology, Towards $100 \mathrm{GHz}$ Logic ed. M. Rodwell

Vol. 22: Topics in High Field Transport in Semiconductors eds. K. F. Brennan and P. P. Ruden

Vol. 23: Oxide Reliability: A Summary of Silicon Oxide Wearout, Breakdown, and Reliability ed. D. J. Dumin

Vol. 24: CMOS RF Modeling, Characterization and Applications eds. M. J. Deen and T. A. Fjeldly

Vol. 25: Quantum Dots eds. E. Borovitskaya and M. S. Shur

Vol. 26: Frontiers in Electronics: Future Chips eds. Y. S. Park, M. S. Shur and W. Tang

Vol. 27: Intersubband Infrared Photodetectors ed. V. Ryzhii

Vol. 28: Advanced Semiconductor Heterostructures: Novel Devices, Potential Device Applications and Basic Properties eds. M. Dutta and M. A. Stroscio

Vol. 29: Compound Semiconductor Integrated Circuits ed. Tho T. Vu

Vol. 30: Terahertz Sensing Technology - Vol. 1 Electronic Devices and Advanced Systems Technology eds. D. L. Woolard, W. R. Loerop and M. S. Shur

Vol. 31: Advanced Device Modeling and Simulation ed. T. Grasser 
Selected Topics in Electronics and Systems - Vol. 32

\title{
TERAHERTZ SENSING TECHNOLOGY
}

\section{Volume 2: Emerging Scientific Applications \& Novel Device Concepts}

\author{
Editors \\ Dwight L Woolard \\ US Army Research Laboratory \\ William R Loerop \\ US Army Soldier Biological and Chemical Command

\section{Michael S Shur} \\ Rensselaer Polytechnic Institute
}




\section{Published by}

World Scientific Publishing Co. Pte. Ltd.

5 Toh Tuck Link, Singapore 596224

USA office: Suite 202, 1060 Main Street, River Edge, NJ 07661

UK office: 57 Shelton Street, Covent Garden, London WC2H 9HE

\section{British Library Cataloguing-in-Publication Data}

A catalogue record for this book is available from the British Library.

\section{TERAHERTZ SENSING TECHNOLOGY}

\section{Volume 2: Emerging Scientific Applications and Novel Device Concepts}

Copyright $\odot 2003$ by World Scientific Publishing Co. Pte. Ltd.

All rights reserved. This book, or parts thereof, may not be reproduced in any form or by any means, electronic or mechanical, including photocopying, recording or any information storage and retrieval system now known or to be invented, without written permission from the Publisher.

For photocopying of material in this volume, please pay a copying fee through the Copyright Clearance Center, Inc., 222 Rosewood Drive, Danvers, MA 01923, USA. In this case permission to photocopy is not required from the publisher.

ISBN 981-238-611-4

Editor: Tjan Kwang Wei

This book is printed on acid-free paper. 


\section{PREFACE}

As we begin the new millennium, significant scientific and technical challenges remain within the terahertz $(\mathrm{THz})$ frequency regime and they have recently motivated an array of new research activities. Indeed, the last research frontier in high-frequency electronics now lies in the so-called terahertz (or submillimeter-wave) regime between the traditional microwave and the infrared domains. Today, the terahertz or $\mathrm{THz}$ regime has been broadly defined as the portion of the submillimeter-wavelength electromagnetic (EM) spectrum between approximately $1 \mathrm{~mm}(300 \mathrm{GHz})$ and $100 \mu \mathrm{m}(3 \mathrm{THz})$. While the $\mathrm{THz}$ frequency regime has long offered many important technical advantages (e.g., wider bandwidth, improved spatial resolution, component compactness) and while significant scientific interest in $\mathrm{THz}$ frequency science and technology has existed since the early 1900 's, the solid-state electronics capability at $\mathrm{THz}$ frequencies today remains extremely limited from a basic signal source and systems perspective (i.e., the highest output power is less than or on the order of milliwatts). The relatively limited development of semiconductor-based electronic circuits within the $\mathrm{THz}$ band may be attributed to the confluence of two fundamental factors. First, very challenging development and engineering problems are present in this quasi-optical regime where EM wavelength is on the order of component size. Second, the practical and scientific applications of this shorter-wavelength microwave region, where the atmospheric propagation paths are extremely attenuating, have been previously restricted to a few specialized fields (e.g., molecular spectroscopy for Earth, planetary and space science). Furthermore, engineering efforts to extend conventional three-terminal semiconductor devices upward from millimeter-wave as well as separate efforts to extrapolate traditional solid-state laser technology down from the far infrared have been prohibited due to fundamental physical factors associated with the respective device technologies. Indeed, electron velocities are not high enough to extend the operational of conventional transit-mode transistors (e.g., HFET's and HBT's) into the THz range even for devices with deep submicron dimensions. Alternatively, when the laser end of the spectrum is considered, one finds that the energies of photonic transitions corresponding to the terahertz range are small compared to the thermal energies at room or elevated temperatures (one terahertz corresponds to a $4.14 \mathrm{meV}$ photon energy and to approximately 300 micron wavelength in free space) and effects, such as free carrier generation, plague these devices.

Fortunately, two-terminal semiconductor devices (e.g., Schottky and Heterostructure Barrier Varactor and Schottky mixers) utilize charging effects very near the contact interface and are inherently faster than transistor devices. Hence, two-terminal transportbased semiconductor devices long ago emerged as the key technology for the generation, amplification and detection of electrical signals at submillimeter-wave frequencies. However, the overall performance (e.g., power and efficiency) of even state-of-the-art two-terminal technologies suffers as they are extended for operation high into the $\mathrm{THz}$ band. This longstanding limitation in $\mathrm{THz}$ electronic technology, along with the excessive cost of instrumentation, have certainly been major stumbling blocks to new scientific inquiries at $\mathrm{THz}$ frequencies and has most probably prevented the spread of $\mathrm{THz}$ science and technology related issues to the broader scientific and engineering communities. Recent advances in nanotechnology, molecular chemistry and biological science have already begun to chart the course for new and important applications of $\mathrm{THz}$ electronics in the coming twenty-first century. In fact, the growing interest in the precise detection, identification and characterization of very small organic and inorganic systems 
has already begun to emphasize the future value of a robust $\mathrm{THz}$-frequency sensing science and to establish it as an important driver for the rapid advancement of electronics technology at $\mathrm{THz}$ frequency. These new and exciting sensing applications only provide added motivation for realizing a practically useful $\mathrm{THz}$ electronics technology, which has long been recognized to offer much to conventional electronic application areas such as extended bandwidth for special scenario communications (i.e., short-range, networked and satellite) and significantly enhanced signal processing power.

During the last few years, major research programs have emerged within the U.S. Army and the Department of Defense (DoD) that have been focused on advancing the state-ofthe-art in THz-frequency electronic technology and on investigating novel applications of THz-frequency sensing. These basic programs grew out of small seed efforts supported by a number of agencies including the U.S. Army Research Laboratory (ARL), the U.S. Soldier Biological and Chemical Command (SBCCOM) and the Air Force Office of Scientific Research (AFOSR). More recently, these efforts have been intensified and propagated primarily from the support of a Defense Advanced Research Project Agency (DARPA) Program on "Terahertz Technology for Sensing and Satellite Communications" and a Multidisciplinary University Research Initiative (MURI) Program on "Sensing Science and Electronic Technology at THz Frequencies" that is managed out of the Army Research Office (ARO) of ARL. One of the main catalysts for these programs is associated with the idea of using the fundamental interactions of $\mathrm{THz}$ radiation at the molecular level for sensing and characterizing chemical and biological (CB) agents. The science and technology emerging from these programs has potentially important ramifications to such areas as CB defense, biomedical applications and molecular science. Historically, the U.S. DoD has been instrumental in establishing the basic foundations for many important endeavors in science and engineering and recent DoD support for $\mathrm{THz}$ electronics is once again playing a major role in promoting an interest among the broader international scientific and engineering community. Indeed, there has been a steadily growing interest among these communities in the unique challenges associated with developing a robust electronics technology and with developing a detailed understanding of $\mathrm{THz}$-frequency sensing science.

This growing wave of research and development has already started to shrink the "THz gap" through recent advances in both photonic and electronic technology. On the photonic technology side, the most prominent recent development has been the emergence of relatively high power $\mathrm{THz}$ quantum cascade lasers developed by Ruedeger, Köhler and Alessandro Tredicucci of the Scuola Normale Superiore in Pisa and colleagues in Turin and Cambridge ${ }^{1}, 2$ (see also earlier work of the Swiss group ${ }^{3}$ This device used 1500 alternating layers of gallium arsenide and aluminum gallium arsenide illustrating dramatic improvements in sophistication of modern semiconductor materials growth technology. This device $(1.5 \mathrm{~mm}$ long and $0.2 \mathrm{~mm}$ wide) emitted $2 \mathrm{~mW}$ at 4.4 THz (wavelength of $67 \mu \mathrm{m}$ ) in single mode operation at $50 \mathrm{~K}$. On the electronic

\footnotetext{
${ }^{1}$ http://physicsweb.org/article/news/6/5/5

${ }^{2}$ http://optics.org/articles/news/8/5/16/1

${ }_{3}^{3}$ Stéphane Blaser, Michel Rochat, Lassaad Ajili, Mattias Beck, Jérôme Faist, H. Beere, A. Davis, E. Linfield, and D. Ritchie, "Terahertz interminiband emission and magnetotransport measurements from a quantum cascade chirped superlattice", Physica E 13, $854(2002)$.
} 
technology side, recent progress in planar integration of two-terminal multipliers and in engineering photomixer technology is defining the new state-of-the-art in solid-state electronic sources and new concepts such as ballistic tunneling devices and plasma waves electronics offer promise for considerable potentials improvements in the future.

The recent plethora of new research activities associated with $\mathrm{THz}$-frequency science and technology has motivated the organization of this book. This two-volume book has been structured to stand as an up to date and detailed reference for the new $\mathrm{THz}$-frequency technological advances that are emerging across a wide spectrum of sensing and technology areas. The first volume (see Terahertz Sensing Technology, Vol. 1 Electronic Devices \& Advanced Systems Technology) emphasized the ongoing efforts to establish a practical and useful components and system base within the $\mathrm{THz}$ regime. This second volume focuses on endeavors that possess higher risk, and much higher payoff, such as innovative applications in sensing, and new emerging device concepts. Hence, the full significance of these research subjects remains to be fully understood and established in many cases.

This second volume of the book presents cutting edge results in two primary areas: (1) research that is attempting to establish $\mathrm{THz}$-frequency sensing as a new characterization tool for chemical, biological and semiconductor materials, and (2) theoretical and experimental efforts to define new device concepts within the "THz gap."

Chapter 1 of this volume begins with an overview of $\mathrm{THz}$-frequency spectroscopic sensing of biological materials by Prof. Tatiana Globus and co-workers. Here, the focus is on spectral measurements of DNA (and related biological materials) that are providing new insight into the microscopic mechanisms associated with the interactions between $\mathrm{THz}$ radiation and species-specific phonon modes. This is a very appropriate chapter to begin this book as it provides an interesting and new scientific basis for the development of future bio-sensing technologies. In Chapter 2, Choi et. al. report on new efforts to utilize broadband $\mathrm{THz}$-frequency reflection and transmission spectra as a starting point for distinguishing concealed threats in envelops and on personnel. Here, thermal modulation is demonstrated as an effective tool to induce changes to the microwave reflections and points to a new way for gaining qualitative chemical and biological spectra from broadband $\mathrm{THz}$ systems. In Chapter 3, Markelz and Whitmire provide results from $\mathrm{THz}$ time-domain spectroscopy performed on biomolecules to determine applicability of the technique for chemical and conformational identification. While this research did not reveal distinct normal modes that could be used for identification, it did demonstrate a non-destructive method to rapidly quantify the conformational flexibility of biomolecular species through the FIR response. This research also suggests a potential approach for evaluating cycling times and response time due to mutagenisis, which will be pursued further in future research investigations. Chapter 4 is authored by Federici and Grebel and describes the use of $\mathrm{THz}$, Raman and IR spectroscopy for the indirect assessments of the electronic characteristics of nanocomposites. Here, $\mathrm{THz}$ spectroscopy was shown to provide insight into the electron mobility in semiconductive and conductive nanostructures that are suitable for electronic, electro-optic and nonlinear optical applications. Prof. Elliott Brown gives a unique and detailed engineering presentation in Chapter 5 on the subject of Terrestrial Millimeter-wave and $\mathrm{THz}$ remote sensing. This excellent treatment of remote sensing addresses all the fundamental principles behind system architectures and overviews all the critical aspects needed for designing a $\mathrm{THz}$ frequency remote sensing system. Indeed, this is an excellent blueprint for anyone who is 
interested in designing and developing a system for the remote detection of chemical and biological agents and stands as an important and extremely useful technical reference. In Chapter 6, Solomon and coworkers explore the prospects of developing a laser operating in the $\mathrm{THz}$ regime using a quantum dot (QD) gain medium. This general device concept holds promise because it overcomes phonon losses in the device active region and is conducive to some $\mathrm{THz}$ laser cavity designs. The research presented in this chapter shows that QDs can be selectively placed in whispering-gallery architectures, while maintaining good QD optical quality. This approach suggests an exciting new opticalbased source technology. Allen and Scott review the physics of $\mathrm{THz}$-frequency transport in quantum structures in Chapter 7 and evaluate electrically-biased superlattices for their potential as terahertz gain medium. This work demonstrates that embedding superlattice devices in quasi-optical arrays and integrating them into terahertz cavities makes it possible to measure the dynamical conductance of quantum structures. This work sheds new light on fundamental issues related to utilizing gain in superlattice structures. The next two chapters in Volume 2 consider issues related to the theory and modeling of $\mathrm{THz}$-frequency oscillations in quantum structures. In Chapter 8, Woolard and coworkers present an advanced theory of electronic instability in tunneling nanostructures. Here, new theoretical concepts and models are presented for the study of one-dimensional tunneling devices that possess the potential for exhibiting self-oscillations at $\mathrm{THz}$ frequencies. This chapter stands as an excellent reference for the study of time-dependent quantum mechanical effects and nonequilibrium phenomena (e.g., multi-band transport). Grubin and Buggeln present results on Wigner-function based simulations in Chapter 9 that can be used to address the effects of device-circuit interactions for quantum devices operating at very fast switching times. This work lays an important foundation for the future simulation of self-sustained $\mathrm{THz}$ oscillations in quantum-based circuits. Volume 2 concludes with Chapter 10 where Plusquellic and coworkers present scientific results generated from a continuous-wave linear-absorption spectroscopic system that utilizes solid-state photomixers as its source. Here, the system was applied to the investigation of biomolecules in polyethylene matrices and to line-shape studies of HF for diagnostics of semiconductor etching plasmas. This chapter directly demonstrates the scientific and commercial potential of THz-frequency systems and is a very appropriate conclusion to Volume 2.

The editors would like to thank all the authors for their fine contributions to this outstanding review of "Terahertz Sensing Technology". Both Volume 1 on Electronic Devices \& Advanced Systems Technology, and Volume 2 on Emerging Scientific Applications \& Novel Device Concepts, will be useful for technologists, scientists, engineers, and graduate students who are interested in the development of terahertz technology for sensing applications. The book can also be used as a textbook for graduate and senior undergraduate courses on terahertz electronics and as an additional reference text for courses in semiconductor physics, materials science, and electronic device design. 


\section{CONTENTS}

Preface

THz-Frequency Spectroscopic Sensing of DNA and Related Biological Materials

T. Globus, D. Woolard, M. Bykhovskaia, B. Gelmont,

L. Werbos, and A. Samuels

Spectroscopy with Electronic Terahertz Techniques for Chemical and Biological Sensing

M. K. Choi, K. Taylor, A. Bettermann, and D. W. van der Weide

Terahertz Applications to Biomolecular Sensing

A. G. Markelz and S. E. Whitmire

Characteristics of Nano-Scale Composites at THz and IR Spectral Regions J. F. Federici and H. Grebel

Fundamentals of Terrestrial Millimeter-Wave and THz Remote Sensing E. R. Brown

Terahertz Emission using Quantum Dots and Microcavities

G. S. Solomon, Z. Xie, and M. Agrawal

Terahertz Transport in Semiconductor Quantum Structures

S. J. Allen and J. S. Scott

Advanced Theory of Instability in Tunneling Nanostructures

D. L. Woolard, H. L. Cui, B. L. Gelmont, F. A. Buot, and P. Zhao

Wigner Function Simulations of Quantum Device-Circuit Interactions

H. L. Grubin and R. C. Buggeln

Continuous-Wave Terahertz Spectroscopy of Plasmas and Biomolecules

D. F. Plusquellic, T. M. Korter, G. T. Fraser, R. J. Lavrich,

E. C. Benck, C. R. Bucher, A. R. H. Walker, and J. L. Domenech 